\title{
A functional calculus for continuous affine operators
}

\section{J.J. Koliha}

In the Appendix to a recent paper by J.J. Kol iha and A.P. Leung (Math. Ann. 216 (1975), 273-284), a functional calculus for continuous affine operators was constructed on the basis of the Taylor-Dunford calculus. This calculus applied only to functions defined and analytic in an open set containing the spectrum of an operator and the point $\lambda=1$. In the present paper I examine the affine resolvent, and develop independently a more general calculus applicable to functions which are analytic in any open neighbourhood of the spectrum of an affine operator.

Let $X$ be a complex Banach space. An operator $A: X \rightarrow X$ is affine if $A(\alpha x+(1-\alpha) y)=\alpha A x+(1-\alpha) A y$ for all $x, y \in X$ and all complex $\alpha$. The trace of $A$ is the linear operator $A^{\#}$ on $X$ defined by

$$
A^{\#} x=A x-A 0, \quad x \in X .
$$

PROPOSITION 1. Let $A, B$ be affine operators on $X$, and let $\lambda, \mu$ be complex numbers. Then:

(i) A is continuous iff $A^{\#}$ is continuous;

(ii) $(\lambda A+\mu B)^{\#}=\lambda A^{\#}+\mu B^{\#},(A B)^{\#}=A^{\#} B^{\#}$;

(iii) if $A$ is bijective, then the inverse $A^{-1}$ is affine, and $\left(A^{-1}\right)^{\#}=\left(A^{\#}\right)^{-1}$;

Received 22 December 1975. 
(iv) A is bijective iff $A^{\#}$ is bijective;

(v) if $A$ is continuous and bijective, its inverse $A^{-1}$ is continuous.

The proof is omitted.

PROPOSITION 2. The set $A(X)$ of all continuous affine operators on $X$ is a Banach space under the norm

$$
\|A\|=\|A O\|+\left\|A^{\#}\right\| \text {. }
$$

The norm topology of $A(X)$ coincides with the topology of uniform convergence on bounded subsets of $X$.

The proof is omitted.

We note that $A(X)$ is a near algebra with the unit $I$, satisfying the laws

$$
(A+B) C=A C+B C, \quad(\alpha A) B=\alpha(A B) .
$$

Furthermore,

$$
\begin{aligned}
& C(A+B) x=(C A+C B) x-C O, \\
& A(\alpha B) x=\alpha(A B) x+(1-\alpha) A O .
\end{aligned}
$$

For any operator $A \in \mathrm{A}(X)$, we define the resolvent set $\rho(A)$ of $A$ as the set of all complex $\lambda$ such that the operator $\lambda I-A$ is bijective; the spectrum $\sigma(A)$ is the complement of $\rho(A)$ in the complex plane. (This definition differs from the one given in [3], where the point $\lambda=1$ was adjoined to $\sigma(A)$ when $A$ was non-linear.) In view of Proposition 1 ,

$$
\rho(A)=\rho\left(A^{\#}\right), \sigma(A)=\sigma\left(A^{\#}\right) .
$$

It follows from [2, pp. 123-125] that the resolvent set is open, and that the spectrum is non-empty and compact. The spectral radius $r(A)$ of $A \in \mathrm{A}(X)$ is the number $r(A)=\sup \{|\lambda|: \lambda \in \sigma(A)\}$.

For $A \in A(X)$, the function $R(\lambda ; A)=(\lambda I-A)^{-1}$ defined for

$\lambda \in \rho(A)$ is the resolvent of $A$. We note that $R(\lambda ; A)^{\#}=R\left(\lambda ; A^{\#}\right)$.

THEOREM 1. For any $A \in \mathrm{A}(X)$ the function $\lambda \mapsto R(\lambda ; A)$ on $\rho(A)$ to $A(X)$ is analytic in the norm topology of $A(X)$. 
Proof. First we show that

$$
R(\lambda ; A) x=R\left(\lambda ; A^{\#}\right)(x+A 0), \lambda \in \rho(A) .
$$

Indeed, applying $\lambda I-A$ to the vector on the right in (I), we get $\left(\lambda I-A^{\#}\right) R\left(\lambda ; A^{\#}\right)(x+A O)+(\lambda I-A) 0=x$, and (1) follows.

Choose $\lambda_{0} \in \rho(A)$. For all $\lambda$ in the disc $\left|\lambda-\lambda_{0}\right|<\left\|R\left(\lambda_{0} ; A^{\#}\right)\right\|^{-1}$ the series $\sum_{n=0}^{\infty}\left(\lambda_{0}-\lambda\right)^{n} R\left(\lambda_{0} ; A^{\#}\right)^{n+1}$ converges to $R\left(\lambda ; A^{\#}\right)$ in norm by Theorem 4.7.1 in [2, p. 123]. Consequently,

$$
R(\lambda ; A) x=\sum_{n=0}^{\infty}\left(\lambda_{0}-\lambda\right)^{n} R\left(\lambda_{0} ; A^{\#}\right)^{n+1}(x+A 0)
$$

uniformly on bounded subsets of $X$.

Let $K$ be a compact subset of an open set $\Omega$ in the complex plane. A cycle $\gamma[1, \mathrm{p} .138]$ is a Cauchy cycle with respect to the pair $(\Omega, K)$ if $\gamma$ has a representation as a sum of rectifiable loops in $\Omega \backslash K$, and if the index $n(\gamma, \lambda)=(2 \pi i)^{-1} \int_{\gamma}(\xi-\lambda)^{-1} d \xi$ equals 0 for all $\lambda \in C \backslash \Omega$, and $I$ for all $\lambda \in K$. The existence of such cycle is demonstrated as follows. Let $\varepsilon>0$ be such that $|\mu-\lambda| \geq \varepsilon$ if $\mu \in C \backslash \Omega$ and $\lambda \in K$. Cover the complex plane with a mesh of squares, each of diameter less than $\varepsilon$, and let $\partial S_{1}, \ldots, \partial S_{n}$ be the positively oriented boundary loops of those closed squares $S_{1}, \ldots, S_{n}$ that meet $K$. Then $\gamma=\partial S_{1}+\ldots+\partial S_{n}$ is a desired cycle.

With each operator $A \in A(X)$ we associate the class $F(A)$ of complex valued functions $f$ defined and analytic in an open neighbourhood $\Delta(f)$ of the spectrum $\sigma(A)$. For $f \in F(A)$, the germ [f] is the set of all $g \in \mathrm{F}(A)$ such that $g(\lambda)=f(\lambda)$ for all $\lambda$ in some open neighbourhood of $\sigma(A)$.

Let $f \in \mathrm{F}(A)$ for some $A \in A(X)$. We put $\Omega(f)=\Delta(f) \backslash\{1\}$ if $\lambda=1$ is in the resolvent set of $A$, and $\Omega(f)=\Delta(f)$ otherwise. We define $f_{\#}$ as the unique function analytic in $\Omega(f)$ satisfying 


$$
f(\lambda)=\tau+(\lambda-I) f_{\#}(\lambda), \lambda \in \Omega(f),
$$

where $\tau=\tau_{f, A}$ equals $f(1)$ if $1 \in \sigma(A)$, and 0 if $1 \in \rho(A)$. Finally, define $f_{*}$ on $\Omega(f)$ by

$$
f_{*}=f_{\#}-f \text {. }
$$

If $A \in \mathrm{A}(X)$ and $f \in \mathrm{F}(A)$, we define $f(A) x$ for each $x \in X$ by the formula

$$
f(A) x=\frac{1}{2 \pi i} \int_{\gamma} f(\lambda) R(\lambda ; A) x d \lambda+\frac{1}{2 \pi i} \int_{\gamma} f_{*}(\lambda) R(\lambda ; A) \operatorname{Od\lambda },
$$

where $\gamma$ is any Cauchy cycle with respect to the pair $(\Omega(f), \sigma(A))$.

THEOREM 2. For any $A \in A(X)$ and any $f \in F(A), f(A)$ is a continuous affine operator on $X$ dependent only on the germ $[f]$.

Proof. The map $x \mapsto R(\lambda ; A) x$ is affine, and the correspondence $h \mapsto \int_{\gamma} h$ is linear; so $f(A)$ is affine. Let $\gamma=\sigma_{1}+\ldots+\sigma_{n}$ be a representation of $\gamma$ by loops in $\Omega(f)$, and let

$$
M=\frac{1}{2 \pi} \sum_{j=1}^{n} \sup _{\lambda \in\left|\sigma_{j}\right|}|f(\lambda)|\left\|R\left(\lambda ; A^{\#}\right)\right\| v\left(\sigma_{j}\right)
$$

Noting that $R(\lambda ; A) x_{1}-R(\lambda ; A) x_{2}=R\left(\lambda ; A^{\#}\right)\left(x_{1}-x_{2}\right)$ for all $x_{1}, x_{2} \in X$, we deduce that $\left\|f(A) x_{1}-f(A) x_{2}\right\| \leq M\left\|x_{1}-x_{2}\right\|$, which proves the (Lipschitz) continuity of $f(A)$.

Let $f_{1}, f_{2}$ be members of $F(A)$ belonging to the germ $[f]$. Let $\gamma_{k}$ be a Cauchy cycle with respect to $\left(\Omega\left(f_{k}\right), \sigma(A)\right), k=1,2$. By assumption, there is an open neighbourhood $\Omega$ of $\sigma(A)$ such that $f_{1}(\lambda)=f_{2}(\lambda)$ for all $\lambda \in \Omega$. Choose a Cauchy cycle $\gamma$ with respect to $(\Omega, \sigma(A))$. For $k \in\{1,2\}, \gamma$ is also a Cauchy cycle with respect to $\left(\Omega\left(f_{k}\right), \sigma(A)\right)$, and $n\left(\gamma-\gamma_{k}, \lambda\right)=0$ if $\lambda \vDash \Omega\left(f_{k}\right) \backslash \sigma(A)$. Hence $\gamma-\gamma_{k}$ is a cycle homologous to zero in $\Omega\left(f_{k}\right) \backslash \sigma(A)$. The homology form of Cauchy's Theorem [1, p. 145] implies that $\int_{\gamma_{k}} h_{k}=\int_{\gamma} h_{k}$ for any analytic 
function $h_{k}$ on $\Omega\left(f_{k}\right) \backslash \sigma(A)$ to $X$. If, in addition, $h_{1}$ and $h_{2}$ are equal on $\Omega$, then

$$
\int_{\gamma_{1}} h_{1}=\int_{\gamma} h_{1}=\int_{\gamma} h_{2}=\int_{\gamma_{2}} h_{2} .
$$

The conclusion now follows as $\lambda \mapsto R(\lambda ; A) x$ is analytic in $\rho(A)$ for each fixed $x \in X$ by Theorem 1 .

If $A$ is linear, the second integral in (3) vanishes, and we have

$$
f(A) x=\frac{1}{2 \pi i} \int_{\gamma} f(\lambda) R(\lambda ; A) x d \lambda,
$$

in agreement with the Taylor-Dunford calculus.

THEOREM 3. For any $A \in \mathrm{A}(X)$ and any $f \in \mathrm{F}(A)$,

$$
f(A) x=f\left(A^{\#}\right) x+f_{\#}\left(A^{\#}\right) A O,
$$

where

$$
f\left(A^{\#}\right)=f(A)^{\#}, \quad f_{\#}\left(A^{\#}\right) A O=f(A) 0
$$

Proof. Let $\gamma$ be a Cauchy cycle with respect to the pair $(\Omega(f), \sigma(A))$. The defining formula (3) implies that $f(A) x-f(A) 0$ is equal to the integral

$$
\frac{1}{2 \pi i} \int_{\gamma} f(\lambda)(R(\lambda ; A) x-R(\lambda ; A) 0) d \lambda,
$$

which is seen to be $f\left(A^{\#}\right) x$. Again by (3),

$$
f(A) 0=\frac{1}{2 \pi i} \int_{\gamma} f_{\#}(\lambda) R(\lambda ; A) 0 d \lambda .
$$

Since $R(\lambda ; A) 0=R\left(\lambda ; A^{\#}\right) A 0$ by $(1)$, we get $f(A) 0=f_{\#}\left(A^{\#}\right) A 0$.

A formula closely related to (4) was used in [3] to define the functional calculus for an affine operator $A$, admitting only functions $f$ analytic in an open neighbourhood $\Delta(f)$ of the set $\sigma\left(A^{\#}\right) \cup\{1\}$. For any such $f$ define $f^{\#}$ on $\Delta(f)$ by $f^{\#}(\lambda)=(\lambda-1)^{-1}(f(\lambda)-f(1))$ if $\lambda \neq 1$, and $f^{\#}(1)=f^{\prime}(1)$. The calculus presented in [3] is defined by the 
formula

(4)'

$$
\bar{f}(A) x=f\left(A^{\#}\right) x+F^{\#}\left(A^{\#}\right) A O,
$$

where $f\left(A^{\#}\right)$ and $f^{\#}\left(A^{\#}\right)$ are interpreted in the sense of the TaylorDunford calculus. To prove the consistency of (4) and (4)', we show that for any member $f$ of $F(A)$ whose domain $\Delta(f)$ contains the point $\lambda=1$ we have $f(A) 0=\bar{f}(A) 0$; that is,

$$
f(A) 0=\frac{1}{2 \pi i} \int_{\sigma} f^{\#}(\lambda) R(\lambda ; A) 0 d \lambda,
$$

where $\sigma$ is any Cauchy cycle with respect to $(\Delta(f), \sigma(A))$.

If $I \in \sigma(A)$, then $f^{\#}=f_{\#}$. Suppose that $I \in \rho(A)$, and recall that $\Omega(f)=\Delta(f) \backslash\{I\}$. Choose a Cauchy cycle $\gamma$ with respect to $(\Omega(A), \sigma(A))$, and a Cauchy cycle $\sigma$ with respect to $(\Delta(f), \sigma(A))$. We note that $\gamma$ is also a Cauchy cycle with respect to $(\Delta(f), \sigma(A))$, so that the difference

$$
\frac{1}{2 \pi i} \int_{\sigma} f^{\#}(\lambda) R(\lambda ; A) 0 d \lambda-\frac{1}{2 \pi i} \int_{\gamma} f_{\#}(\lambda) R(\lambda ; A) 0 d \lambda
$$

is equal to

$$
\frac{1}{2 \pi i} \int_{\gamma} f(I)(\lambda-1)^{-1} R(\lambda ; A) 0 d \lambda \text {. }
$$

The last integral vanishes since the integrand is analytic in $\Omega(f)$, and the cycle $\gamma$ homologous to zero in $\Omega(f)$. This result combined with (5) establishes (5)'.

The foregoing argument illuminates our convention that the point $\lambda=1$ be deleted from $\Delta(f)$ when $1 \in \rho(A)$.

To test the formula (3) as a basis for a functional calculus, we prove that for each $x \in X$,

$$
f_{k}(A) x=A^{k} x \text { if } f_{k}(\lambda)=\lambda^{k}, k=0,1, \ldots .
$$

According to the formula (4), this is equivalent to

$$
f_{k}\left(A^{\#}\right) x=A^{\# k} \text { and } f_{k}(A) 0=A^{k_{0}} \text {. }
$$


The first equation in (6) follows from the well known power series expansion for the linear resolvent $R\left(\lambda ; A^{\#}\right)$ (Theorem 4.7 .2 in $[2$, p. 124]). In view of (5)', the second equation in (6) is equivalent to

$$
\frac{1}{2 \pi i} \int_{\sigma}\left(\sum_{j=0}^{k-1} \lambda^{j}\right) R(\lambda ; A) \operatorname{Od} \lambda=A^{k_{0}} \text {, }
$$

where $\sigma$ is any Cauchy cycle with respect to $(C, \sigma(A))$, and where $\sum_{j=0}^{-I}=0$. Proceeding by induction, we obtain

$$
\begin{aligned}
\frac{1}{2 \pi i} \int_{\sigma}\left(\sum_{j=0}^{k} \lambda^{j}\right) R(\lambda ; A) 0 d \lambda & =A^{k} 0+\frac{1}{2 \pi i} \int_{\sigma} \lambda^{k} R\left(\lambda ; A^{\#}\right) A 0 d \lambda \\
& =A^{k} 0+A^{\# k} A 0 \\
& =A^{k+1} 0 .
\end{aligned}
$$

THEOREM 4. Let $A \in A(X)$, let $f, g \in \mathrm{F}(A)$, and let $\alpha, \beta$ be complex numbers. Then:

(i) $\alpha f+\beta g \in F(A)$, and $(\alpha f+\beta g)(A)=\alpha f(A)+\beta g(A)$;

(ii) $f \cdot g \in F(A)$, and

$$
f(A) g(A) x=(f \cdot g)(A) x+(1-\tau) f(A) 0,
$$

where $\tau=\tau_{g, A}$ equals $g(1)$ if $1 \in \sigma(A)$, and 0 if $I \in \rho(A)$;

(iii) if $f$ has the power series expansion $f(\lambda)=\sum_{k=0}^{\infty} \alpha_{k} \lambda^{k}$ valid in an open neighbourhood of $\sigma(A)$, then $f(A)=\sum_{k=0}^{\infty} \alpha_{k} A^{k}$ in the norm of $A(X)$;

(iv) $\sigma(f(A))=f(\sigma(A))$.

Proof. (i) This follows from the defining formula (3) and the identity $(\alpha f+\beta g)_{*}=\alpha f_{*}+\beta g_{*}$.

(ii) If $A$ is linear, we apply the argument given in (5.2.7) [2, p. 169] with $\Gamma$ and $\Gamma^{\prime}$ chosen as follows: let $\Omega=\Omega(f) \cap \Omega(g)$, and let $D$ be a bounded open neighbourhood of $\sigma(A)$ whose closure $\bar{D}$ is 
contained in $\Omega$. Then select $\Gamma$ as a Cauchy cycle with respect to $(D, \sigma(A))$, and $\Gamma^{\prime}$ as a Cauchy cycle with respect to $(\Omega, \bar{D})$. We conclude that

$$
f(A) g(A)=(f \cdot g)(A) .
$$

Let $A$ be affine. In view of Theorem 3 and the preceding result for linear operators, (7) will be established when we show that

$$
f(A) g(A) 0=(f \cdot g)(A) 0+(1-\tau) f(A) 0 \text {. }
$$

Applying (4), the preceding result for linear operators, and part ( $i$ ) of the present theorem, we reduce ( 8 ) to

$$
\left(f \cdot g_{\#}+f_{\#}\right)\left(A^{\#}\right) A O=\left((f \cdot g)_{\#}+(1-\tau) f_{\#}\right)\left(A^{\#}\right) A O ;
$$

this equation holds as $(f \cdot g)_{\#}=f \cdot g_{\#}+\tau f_{\#}$.

(iii) Using the first equation in (6) and the limit passage under the integral sign, we obtain the series expansion

$$
f\left(A^{\#}\right)=\sum_{k=0}^{\infty} \alpha_{k} A^{\# k} \quad \text { (in the operator norm). }
$$

Let $I \in \sigma(A)$. Then $f_{\#}=f^{\#}$, and

$$
f^{\#}(\lambda)=\sum_{k=0}^{\infty} \alpha_{k}\left(\sum_{j=0}^{k-1} \lambda^{j}\right)
$$

uniformly on compact subsets of $\Delta(f)$ by (A9) in [3]. According to the formula (5) and the second equation in (6), $f(A) 0$ is given by

$$
\sum_{k=0}^{\infty} \alpha_{k}\left(\frac{1}{2 \pi i} \int_{\gamma}\left(\sum_{j=0}^{k-1} \lambda^{j}\right) R(\lambda ; A) 0 d \lambda\right)=\sum_{k=0}^{\infty} \alpha_{k} A^{k_{0}}
$$

Let $1 \in \rho(A)$. Then $f_{\#}(\lambda)=(\lambda-1)^{-1} f(\lambda)$ for all $\lambda \in \Delta(f) \backslash\{1\}$, and $f(A) 0$ is equal to

$$
\sum_{k=0}^{\infty} \alpha_{k}\left(\frac{1}{2 \pi i} \int_{Y}(\lambda-1)^{-1} \lambda^{k} R(\lambda ; A) 0 d \lambda\right)
$$

for any Cauchy cycle $\gamma$ with respect to $(\Delta(f) \backslash\{1\}, \sigma(A))$. The integral under the summation sign is equal to 


$$
\frac{1}{2 \pi i} \int_{\gamma}\left(\sum_{j=0}^{k-1} \lambda^{j}\right) R(\lambda ; A) 0 d \lambda+\frac{1}{2 \pi i} \int_{\gamma}(\lambda-1)^{-1} R(\lambda ; A) 0 d \lambda ;
$$

the second integral vanishes, and we have again

$$
f(A) 0=\sum_{k=0}^{\infty} \alpha_{k} A^{k_{0}} .
$$

The result follows from (9) and (10).

(iv) Since $\sigma(f(A))=\sigma\left(f(A)^{\#}\right)=\sigma\left(f\left(A^{\#}\right)\right)$, we can apply the spectral mapping theorem for bounded linear operators [2, p. 171].

Theorem 4 (i), (ii), (iii) extend the correspondingly numbered parts of Theorem Al in [3] to arbitrary members $f, g$ of $\mathrm{F}(A)$. The best result on composite functions seems to be Theorem AI (iv) of [3] which states that

$$
h(f(A))=(h \circ f)(A)
$$

if $f \in \mathrm{F}(A)$ is such that $f(1)=1$, and if $h \in \mathrm{F}(f(A))$. When we relinquish the requirement $f(1)=1$, we can only conclude that $h(f(A))-(h \circ f)(A)$ is a constant operator.

We observe that the operators $f(A), g(A)$ do not commute in general; however, the commutator $[f(A), g(A)]=f(A) g(A)-g(A) f(A)$ is a constant operator, namely

$$
[f(A), g(A)] x=[f(A), g(A)] 0, \quad x \in X .
$$

We conclude the paper with an application.

EXAMPLE. Let $T$ be a bounded linear operator on $X$, and let $y, z \in X$ be given. We show that the differential equation

$$
\frac{d y(t)}{d t}=T y(t)+e^{t} z, y(0)=y,
$$

in the real variable $t$ has a unique solution given by

$$
y(t)=e^{t A} y,
$$

where $A$ is the affine operator defined by $A x=T x+z$.

Clearly, it is enough to prove that 


$$
\frac{d}{d t} e^{t A} y=A e^{t A} y+\left(e^{t}-1\right) z
$$

Put $G(t, \lambda)=e^{t \lambda}$, and define $G(t, A)$ in accordance with (3). Differentiating under the integral sign, and observing that $\partial G_{*} / \partial t=(\partial G / \partial t)_{*}$, we obtain that

$$
\frac{d}{d t} e^{t A}=\frac{\partial G}{\partial t}(t, A)
$$

The result then follows when we find that

$$
\frac{\partial G}{\partial t}(t, A) y=A e^{t A} y+\left(e^{t}-1\right) z
$$

by Theorem 4 (ii) with $f(\lambda)=\lambda$ and $g(\lambda)=e^{t \lambda}$.

\section{References}

[1] Lars V. Ahlfors, Complex analysis: an introduction to the theory of analytic functions of one complex variable, second edition (McGraw-Hill, New York; Toronto, Ontario; London; 1966).

[2] Einar Hille and Ralph S. Phillips, Functional analysis and semigroups (Colloquium Publications, 31, revised edition. Amer. Math. Soc., Providence, Rhode Island, 1957).

[3] J.J. Koliha and A.P. Leung, "Ergodic families of affine operators", Math. Ann. 216 (1975), 273-284.

Department of Mathematics, University of Melbourne,

Parkville,

victoria. 